\title{
Safety and Efficacy of a Thermally Regulated Radiofrequency Home-Device for Rhytide and Laxity Treatment
}

\author{
Judith Hellman1, Hela Goren², R. Stephen Mulholland ${ }^{3 *}$ \\ ${ }^{1}$ New York, USA \\ ${ }^{2}$ Yokneam, Israel \\ ${ }^{3}$ Private Plastic Surgery Practice, Toronto, Canada \\ Email: ${ }^{*}$ info@spamedica.com
}

Received 25 May 2015; accepted 31 July 2015; published 4 August 2015

Copyright (C) 2015 by authors and Scientific Research Publishing Inc.

This work is licensed under the Creative Commons Attribution International License (CC BY).

http://creativecommons.org/licenses/by/4.0/

(c) (i) Open Access

\section{Abstract}

The growth of the medical aesthetic industry is tremendous, and not surprisingly, has been followed by the introduction of new novel technologies for home use. This study focuses on the Silk'n Home Skin Tightening (HST), a home use device that uses a thermally regulated radiofrequency (RF) delivery technology. This technology allows the end point dermal temperatures to be sustained safely for a prolonged period of time, optimizing the non-ablative dermal collagen, elastin and ground substance production. Enhancement of the clinical results of rhytides and laxity improvement are noted. In this study, the HST protocol and technology innovation is outlined. The results of a blinded, independent assessment of photographs before and 1 month after 20 biweekly sessions demonstrated that $96 \%$ of patients achieved an average improvement of 1.6 grades (32\%) in their Fitzpatrick wrinkling and elastosis scale, with no significant complications.

\section{Keywords}

Radiofrequency, Wrinkle Reduction, Skin Tightening, HST

\section{Introduction}

Patient demand for non-surgical, non-invasive and no-downtime wrinkle and laxity reduction procedures has grown dramatically over the past decade. This increased demand has fuelled the growth of new technologies that deliver these non-invasive and non-ablative, anti-aging outcomes. The disadvantages of in-office based treat-

\footnotetext{
"Corresponding author.

How to cite this paper: Hellman, J., Goren, H. and Mulholland, R.S. (2015) Safety and Efficacy of a Thermally Regulated Radiofrequency Home-Device for Rhytide and Laxity Treatment. Journal of Cosmetics, Dermatological Sciences and Applications, 5, 169-175. http://dx.doi.org/10.4236/jcdsa.2015.53020
} 
ments are availability, inconvenience of travel to the clinic for multiple sessions, cost of the treatments, discomfort with the high fluence devices, and risks of pigmentation issues and scars. Using a small low energy homeuse system can alleviate these disadvantages.

The non-ablative rhytide reduction technologies can be divided into a) laser and infrared (IR) devices with single spot size or laser/IR “stampers", and b) radiofrequency (RF) or RF/IR combination with single spot size and "stamping" technologies, both yielding high peak thermal end points. The early prototypes for the "stamping” laser devices included the CoolTouch 1320 nm laser, the Titan for the pure IR "stampers", and Thermage for the "RF stamping" technology. Each of these aforementioned devices had considerable success by heating the dermis to non-ablative temperatures of $45^{\circ} \mathrm{C}-50^{\circ} \mathrm{C}$, while protecting the epidermis from overheating by direct cooling systems or specific pulse configurations. The non-ablative heating resulted in a thermally mediated, micro-inflammatory production of collagen, elastin and ground substances, with a moderate improvement in rhytides and laxity. Due to the short pulse durations and short exposure time of the dermis to the non-ablative temperatures, multiple pass and multiple session protocols evolved. However, the high peak temperatures and short pulse durations of these "stampers" led to a high and often unacceptable level of patient discomfort and clinically inconsistent rhytide improvement outcomes.

In attempt to overcome these limitations of the early generation non-ablative skin heating devices, there evolved a class of continuous radio frequency (RF) thermal skin heaters that necessitated moving the applicator around on the skin surface, while the RF heated the epidermal-dermal tissues. These "moving” RF heating systems include Accent (Alma Lasers), Exilis (Aesthetic Precision) and Venus Freeze (Venus Concept) and have grown in popularity to dominate the market, as they sustain the dermal matrix to elevated thermal temperatures for prolonged periods of time, leading to more consistent rhytide and laxity results. There are several monopolar, bipolar and multipolar RF devices in the market, however, this current generation of “moving” RF applicators does not have thermal feedback control and can lead to "thermal spikes", patient discomfort and compromised outcomes or patient compliance issues.

This paper introduces novel technology advancement and "moving” RF soft tissue heating application, which is called the Silk'n HST by HomeSkinovations Ltd., Yokneam, Israel. It introduces for the first time, an RF device that senses skin temperatures and controls the deliverd RF energy, allowing a prolonged, sustained, safe and efficacious dermal heating.

The effects of dermal heating are well recognized to include immediate effects on collagen structure with stimulation of neocollagenesis [1]. These changes can help reduce the appearance of fine wrinkles and enhance skin tightening. RF energy has been used in medicine for several decades. It heats tissue through intermolecular motion, molecular friction and the induction of rotational movement in water molecules. During the course of treating wrinkles and laxity, the treatment creates enough thermal effect to induce collagen, elastin and ground substance remodeling with no ablative thermal damage of the epidermis or dermis, nor thermal reduction of the subcutaneous adipose tissue.

This study was performed in order to evaluate the efficacy and safety of the HST home-use device, based on an innovative, noninvasive highly thermally regulated RF heating technology, intended for the reduction of rhytides and for skin tightening

\section{Materials and Methods}

\subsection{Study Design}

This study is a multicenter study, performed in 3 clinics in USA and Canada. Fifty patients were treated on 3 facial areas-2 cheeks and forehead; altogether 150 treatment sites. Twenty bi-weekly treatment sessions during 10 weeks were conducted at home, following $1^{\text {st }}$ session of instruction in the clinics. Follow up session was 1 month after the last treatment.

\subsection{Device Description}

The HST device consists of two side electrodes and one central electrode (Figure 1). RF current alternates between the side electrodes and the central electrode pins, confined to the dermis and superficial subdermal layer. There is an impedance sensor and a skin surface thermal sensor built into the applicator. Addition of these sensors allows for an innovative and valuable thermal treatment, allowing the skin to be treated at the desired ther- 


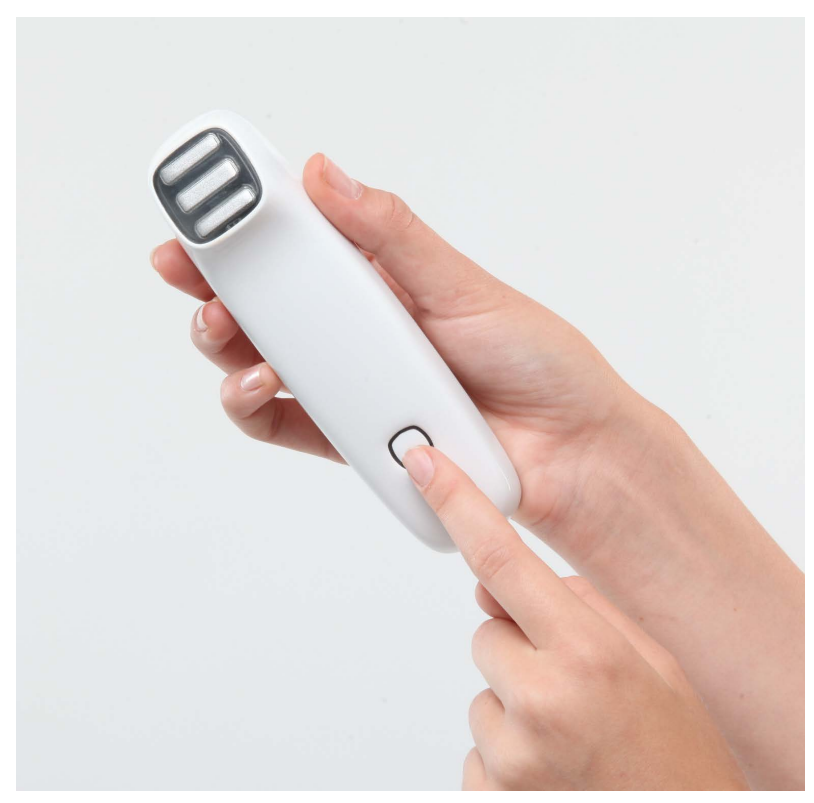

Figure 1. The HST home-device.

mal end point for prolonged periods of time, minimizing "hot spots" and patient discomfort, optimizing dermal matrix response, wrinkle reduction, and laxity improvement. There are 5 power levels that are chosen by the operator according to comfort. Levels 3 - 5 are emitting 5 watts, 10 watts, and 15 watts RF, respectively. In addition there is heating via a red spectrum LED. When the epidermal temperature reaches the end point temperature $\left(41^{\circ} \mathrm{C}-43^{\circ} \mathrm{C}\right.$ in levels $3-5$, respectively), the device senses this temperature and the RF energy is inactivated. Once the temperature falls below the end point temperature, the RF is turned back on again. This cycle will continue as long as the operator wishes to maintain this soft tissue thermal end point. Thus, temperature end point is achieved quickly, well controlled and safely sustained. Additional safety features include the ability of the impedance sensors to detect when the dermal temperature is rising too quickly and the dermal impendence drops suddenly, resulting in the RF energy to be shut-off automatically. Conversely, if the electrodes stray off the skin, the impedance rises too high and the RF is shut-off (limiting RF arching or poor contact discomfort).

\subsection{Subjects}

53 subjects (44 females and 9 males) were enrolled in the study. Of all 53 subjects, 50 subjects (94\%) completed the study course and received all 20 treatments and attended the 1 month follow-up visit. Three subjects withdrew from the study because they were not compliant with the treatment protocol. Subjects' age was between 36 77 (average $55.5 \pm 10.8$ ) with Fitzpatrick skin type I to VI (Table 1). Subjects were enrolled in the study after meeting all inclusion/exclusion criteria and providing a signed Informed Consent Form. Subjects' wrinkle and rhytide appearance were classified according to Fitzpatrick wrinkling and elastosis scale (Table 2) before and after treatment. Enrolled subjects were classified as having mild or moderate wrinkling and elastosis score according to Fitzpatrick scale. Exclusion criteria included scarring, inflammation or infection of the area to be treated, history of skin disorders, keloids, abnormal wound healing, pregnancy or lactating, subjects with current or history of malignancy, implants or a pacemaker device, Botox/HA/collagen/fat injections or other augmentation methods with bio-material in the last six months, poorly controlled endocrine disorders as diabetes or thyroid dysfunction, severe concurrent conditions such as cardiac disorders, epilepsy, uncontrolled hypertension and liver and kidney disorders, patients with history of diseases stimulated by heat, such as recurrent Herpes Simplex in the treated area, facial resurfacing, deep chemical peeling within the last 3 months, or history of bleeding coagulopathies.

\subsection{Treatment Protocol}

The first session took place in the clinics and the subjects were trained and performed the first treatment at the 
Table 1. Subject demographic.

\begin{tabular}{ccc}
\hline Age & Gender & Skin type \\
\hline $55.5 \pm 10.8$ & 8 males & $3.3 \pm 1.3$ \\
\hline
\end{tabular}

Table 2. Fitzpatrick wrinkling and elastosis scale [2].

\begin{tabular}{cccc}
\hline Class & Wrinkling & Score & \\
\hline I & Fine wrinkles & $\mathbf{1 ~ - 3}$ & Mild: fine texture changes with subtly accentuated skin lines. \\
II & $\begin{array}{c}\text { Fine to moderate depth wrinkles } \\
\text { Moderate number of lines }\end{array}$ & $\mathbf{4 - 6}$ & $\begin{array}{c}\text { Moderate: distinct popular elastosis } \\
\text { (individual papules with yellow translucency) and dyschromia. }\end{array}$ \\
III & $\begin{array}{c}\text { Fine to deep wrinkles, numerous lines } \\
\text { With or without redundant skin folds }\end{array}$ & $\mathbf{7 - 9}$ & $\begin{array}{c}\text { Severe: multipapular and confluent elastosis } \\
\text { (thickened, yellow and pallid) approaching with cutis rhomboidalis. }\end{array}$ \\
\hline
\end{tabular}

investigators' offices under the supervision of trained nurses. Treatment power level (5 - 15 W) was adjusted to each patient according to tolerance and skin response. All patients could tolerate $15 \mathrm{~W}$ at level 5 . Prior to initiating the study, the treated areas were photographed in a standardized method, using high-resolution digital photography in order to allow comparison and assessment of wrinkle and laxity improvement at study conclusion.

All subsequent treatments were performed at home by the patients. They were instructed to call the clinic for any query that they may have or for any change in response that could be classified as an adverse event. Subjects came to the clinics after 10 bi-weekly sessions to evaluate the interim results and the treatment technique.

The treatment area was cleaned thoroughly with skin cleanser to remove any makeup, perfume, oils or other cosmetics. The face was divided into 3 treatment zones as shown in Figure 2, including the two zones of cheek-jaw line-lower eyelid and the brow-temporal zone, and the forehead. A thin layer of water based gel was applied on the intended treatment area. The treatment technique calls for complete contact with the skin and constant slow circular movements, figure of eights, or in lines throughout the treatment zone. Each zone was treated for 10 minutes, in areas that were sensitive or boney the treatment time could be decreased to seven minutes. The treatment area was monitored thoroughly during the treatment and assessed for any potential adverse events.

Immediately after each treatment the treated area was visually assessed for skin responses and moisturizer was applied. In addition, patients were asked to rate the pain level they felt during treatment, on a 10-point linear analog pain scale.

\subsection{Safety Measures}

The safety of the procedure was evaluated by monitoring the occurrence of potential procedure-related side effects. (e.g. blisters, prolonged discoloration, edema, erythema, hypopigmentation, hyperpigmentation, scars and textural changes).

\subsection{Efficacy Measures}

The study's efficacy end point was evaluated by comparing standardized pre- and post-treatment photographs that were assessed and graded by a blinded independent physician, who is not the study investigator, in each of the 3 clinics. Assessment was based on Fitzpatrick wrinkling and elastosis scale. Any wrinkle score improvement (downgrade score) following treatment, relative to pre-treatment wrinkle (baseline) score, was considered a "success". Subjects were considered a "success" if they gain at least one degree of facial wrinkle reduction (based on Fitzpatrick scale) following 20 treatments. Study success was declared if at least $90 \%$ of study participants were scored a rhytide improvement success.

\subsection{Statistical Analyses}

Descriptive statistics including mean, standard deviation, and 95\% confidence interval were used to describe treatment efficacy (determined by pre and post Fitzpatrick wrinkling and elastosis score). The mean differences 
of Fitzpatrick Score before treatment and at follow up session were analyzed by a paired-sampled t-test. All statistical data analyses were performed using statistical software (SAS version 9.1).

\section{Results}

No unexpected adverse side effects were detected or reported during the study, including Fitzpatrick dark skin types V and VI. All patients participating in the study reported no significant pain during the treatment.

Photographic analysis of pre-and post treatment of the digital images was conducted by a blinded independent physician on each treatment site separately (left cheek, right cheek, and forehead) (Figure 3). Analysis of the 150 treatment facial sites revealed average improvement in 1.6 (STDV 0.7) Fitzpatrick score in almost all subjects (96\% of 150 treatment sites). 15 treatment sites (10\%) improved in 3 Fitzpatrick grades, 72 (48\%) treatment sites improved in 2 Fitzpatrick grades, 57 treatment sites (38\%) improved in 1 Fitzpatrick grade and only 6 treatment sites (4\%) did not show any improvement. Most subjects (94\%) were satisfied with treatment results.

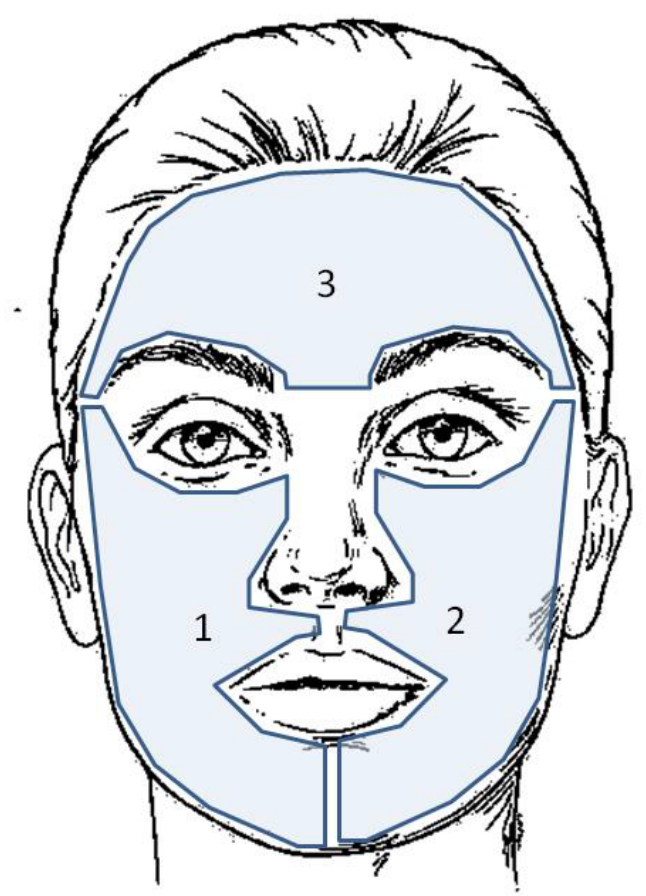

Figure 2. Treatment zones.

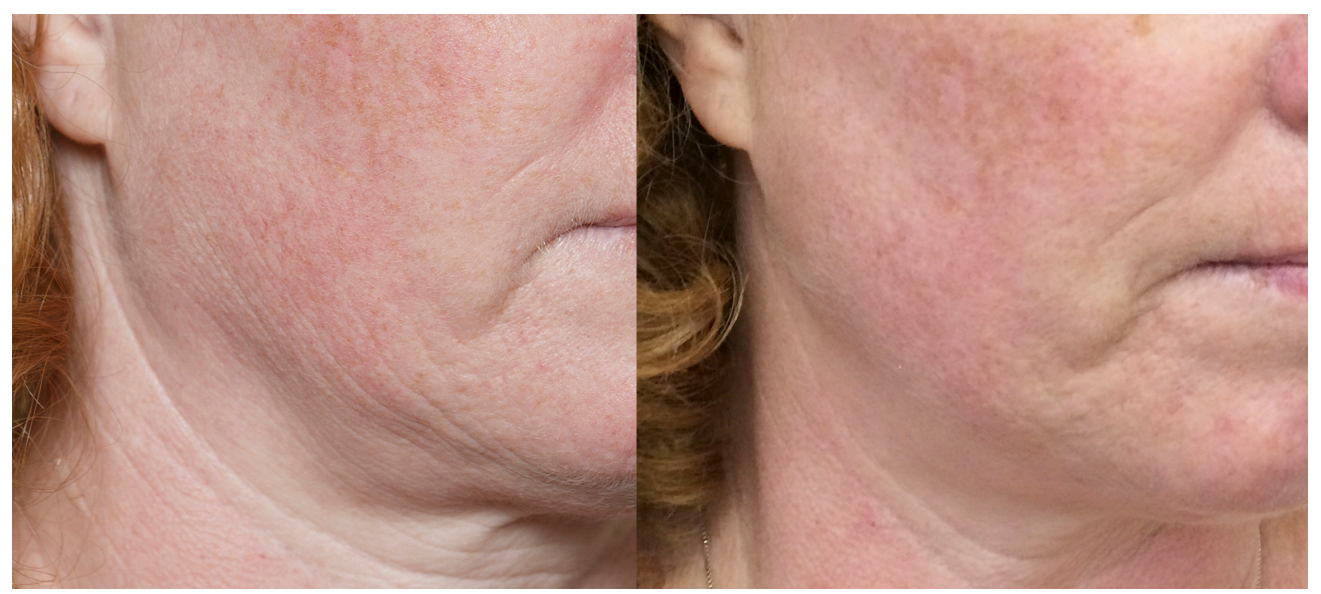

Figure 3. Before and after of lift in the cheek area. 


\subsection{Treatment Safety Assessment}

Post treatment slight erythema was detected in all subjects. The erythema disappeared within one hour without any intervention. In some subjects, post treatment edema (hyperemia) was detected. The edema was resolved within 24 hours, and no treatment was needed. It should be noted that edema and erythema are common transient response following RF treatments and represent a desirable effect.

\subsection{Treatment Efficacy Assessment}

Most patients (94\%) were satisfied from treatment results.

Statistical comparison (using paired t-test) was conducted among the pre treatment Fitzpatrick score (baseline) to Fitzpatrick score obtained at 1 month follow-up. The statistical analysis was conducted by SAS. Score differences were found to be statistically significant while comparing baseline scores to the scores obtained at 1 month follow-up ( $\mathrm{p}<0.001$ ), indicating treatment efficacy. Table 3 and Figure 4 represent averages $( \pm$ STDV) of Fitzpatrick scores given by the blinded independent physician at baseline and at 1 month follow-up. There was no significant correlation between rhytide reduction and skin type; all skin types showed improvement. The results are summarized in Table 3.

\section{Discussion and Conclusions}

The HST home-device, with its thermal control innovation allows the operator to sustain the desired dermal temperatures with exquisite control and comfort, optimizing both the patient experience and non-ablative dermal enhancement. The combination of built in impedance sensors, limiting both high and low values, allows the RF to be cut-off when the dermal temperature is rising too quickly (low impedance cut-off) or when there is poor electrode contact (high impedance cut-off), making the treatment very safe. In addition, the thermal sensor and automatic feedback loop allows the operator and system to tightly regulate the dermal temperature and sustain the thermal end point.

The data reported in this study demonstrate that the HST home-device offers a safe and effective in-home noninvasive technique to improve the appearance of age-related wrinkles and elastosis.

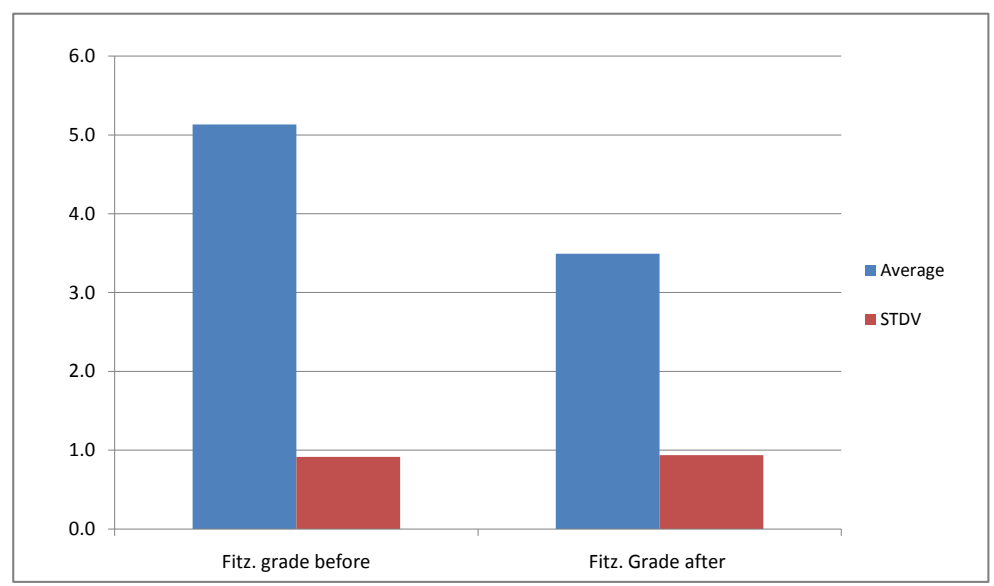

Figure 4. Average $( \pm$ STDV) of Fitzpatrick scores before and 1 month after the last treatment.

Table 3. Average ( \pm STDV) of Fitzpatrick scores at baseline and 1 month follow-up (following 20 treatments), and grade reduction, comparing to baseline with statistical results of the comparison.

\begin{tabular}{ccccc}
\hline Score time & Average score & $\begin{array}{c}\text { Grade reduction } \\
\text { (comparing to baseline) }\end{array}$ & $\begin{array}{c}\text { Grade reduction } \\
\text { in \% }\end{array}$ & $\begin{array}{c}\text { Statistical (t-test) } \\
\text { results of the comparison }\end{array}$ \\
\hline Baseline & 5.1 & & & \\
1 month follow-up & $( \pm 0.9)$ & 1.6 & 31.9 & $\mathrm{p}<0.001$ \\
\hline
\end{tabular}


The clinical results of nonablative RF anti wrinkle effects were first reported in the periorbital area [3]. Fitzpatrick and his colleagues demonstrated clinical improvement in periorbital rhytides in $80 \%$ of subjects. In contrast, in 24 patients who underwent a single RF treatment to improve the upper third of the face, only $36 \%$ of the patients' self-assessment reported improvement [4].

In this study, statistical analysis has revealed improvement (downgrade of average 1.6 score according to the Fitzpatrick scale) in almost all subjects. Analysis of study results has revealed statistical significance while comparing baseline score to the scores obtained at 1 month follow-up, following 20 bi-weekly treatments, indicating treatment efficacy. These results may indicate that the treatments have initiated collagen remodeling process that continues after treatment session has been completed. Study success criteria are defined as: at least $90 \%$ of study subjects are considered success. Therefore, it concludes that study success criteria are met.

In a similar device, it has been reported that the use of a RF device is associated with significant pain, and in a small but significant number of cases subcutaneous fat atrophy develops [5]. No subcutaneous fat atrophy is detected externally. All subjects participating in this study have reported no pain during treatment, although the procedure is performed without using any anesthetic agents. Furthermore, no subject considers the procedure intolerable at any stage.

The HST home-device deploys RF power of only $7.5 \%$ of the density emitted by RF devices currently on the market: up to $4.5 \mathrm{~J} / \mathrm{cm}^{2}$. This low energy can't cause complications and therefore is very safe to the user's skin. As a result, the potential risk is minimal.

The results of this study clearly indicate that the HST innovative RF application offers in-home noninvasive, effective, safe and virtually painless wrinkle reduction and skin laxity treatment.

The HST can also be used to treat rhytide and laxity on non-facial skin, such as the neck, back of hands, navel area, or knees. Further studies may demonstrate this versatility.

\section{References}

[1] Sadick, N. and Sorhaindo, L. (2005) The Radiofrequency Frontier: A Review of Radiofrequency and Combined Radiofrequency Pulsed Light Technology in Aesthetic Medicine. Facial Plastic Surgery, 21, 131-138. http://dx.doi.org/10.1055/s-2005-872414

[2] Goldman, P. and Fitzpatrick, E. (1999) Cutaneous Laser Surgery-The Art and Science of Selective Photothermolysis. Mosby, St. Louis, 377.

[3] Fitzpatrick, R., Geronemus, R., Goldberg, D., et al. (2003) Multicenter Study of Noninvasive, Radiofrequency for Periorbital Tissue Tightening. Lasers in Surgery and Medicine, 33, 232-242. http://dx.doi.org/10.1002/lsm.10225

[4] Bassichis, B.A., Dayan, S. and Thomas, J.R. (2004) Use of Nonablative Radiofrequency Device to Rejuvenate the upper One-Third of the Face. Otolaryngology-Head and Neck Surgery, 130, 397-406. http://dx.doi.org/10.1016/j.otohns.2003.09.034

[5] Biessman, B.S. (2005) Radiofrequency Devices. Monopolar vs Bipolar vs Radiofrequency plus Laser; Indications; Treatment Approaches; Novel Applications; Results. In: Arndt, K.A., Dover, J.S. and Anderson, R.R., Eds., Controversies and Conversations in Laser and Cosmetic Surgery, Symposium Proceedings, Denver, CO. 